\title{
Ideals of Homogeneous Polynomials
}

\author{
by
}

Richard M. Aron and Pilar RuedA

\begin{abstract}
Given a surjective ideal of operators, we undertake a new general procedure to construct an ideal of polynomials. The relation with the ideal of polynomials obtained by the well-known method of composition is established.
\end{abstract}

2010 Mathematics Subject Classification: Primary 46G20; Secondary 46B20, 46G25. Keywords: operator ideals, homogeneous polynomials, symmetric tensor products.

\section{$\S 1$. Introduction}

Ever since Pietsch [16] initiated a research program on ideals of nonlinear operators, many authors have turned their attention to studying ideals of multilinear mappings. The transition from the multilinear setting to homogeneous polynomials occurred naturally.

Several strategies have been developed when trying to generalize ideals of operators to polynomials. A standard procedure is to mimic the property that characterizes the operators belonging to some operator ideal. For instance, (weakly) compact $m$-homogeneous polynomials are defined as those that map bounded sets to relatively (weakly) compact sets, copying the definition of a (weakly) compact operator. This technique, although useful in order to get the first basic examples, lacks the generality required in order to treat polynomial ideals in a systematic way. In this paper we undertake a study of a general method to generate an ideal of polynomials from an operator ideal $\mathcal{I}$. To accomplish this task, we introduce the concept of local $\mathcal{I}$-boundedness. We prove that the set of homogeneous polynomials that are locally $\mathcal{I}$-bounded form a Banach ideal of polynomials when endowed

Communicated by H. Okamoto. Received September 28, 2011. Revised February 15, 2012.

R. M. Aron: Department of Mathematical Sciences, Kent State University,

Kent, Ohio 44242, USA;

e-mail: aron@math.kent.edu

P. Rueda: Departamento de Análisis Matemático, Universidad de Valencia,

Doctor Moliner 50, 46100 Burjasot (Valencia), Spain;

e-mail: pilar.rueda@uv.es

(C) 2012 Research Institute for Mathematical Sciences, Kyoto University. All rights reserved. 
with a naturally defined norm. We consider only a particular case of the wellknown method called composition ideal to generate polynomial ideals from a given operator ideal $\mathcal{I}$. This method consists in composing continuous $m$-homogeneous polynomials with operators belonging to $\mathcal{I}$. The composition ideal is formed by all polynomials $P$ that factor as $P=T \circ Q$, where $Q$ is a polynomial and $T$ belongs to the ideal $\mathcal{I}$ (see Section 2 for a formal description). From [19] it follows that compact and weakly compact polynomials form a composition ideal of polynomials. In [3] the present authors proved that $p$-compact homogeneous polynomials (mapping bounded sets to relatively $p$-compact sets) also form a composition ideal of polynomials.

By [19] any continuous $m$-homogeneous polynomial $P$ is associated to a unique linear operator $P_{L}$ called the linearization of $P$. This linearization yields another way to generate ideals of polynomials, by simply considering those continuous $m$ homogeneous polynomials whose linearization belongs to a given operator ideal $\mathcal{I}$. The polynomial ideal generated in this way turns out to be the same as the composition ideal of polynomials (see [6]).

After reviewing some preliminaries in Section 2, given a Banach operator ideal $\mathcal{I}$, in Section 3 we introduce the concept of local $\mathcal{I}$-boundedness and we construct the ideal $\mathcal{P}_{\mathcal{I}}$ of $\mathcal{I}$-bounded polynomials. We prove that whenever $\mathcal{I}$ is stable under the formation of symmetric tensor products then any continuous $m$-homogeneous polynomial maps $\mathcal{I}$-bounded sets to $\mathcal{I}$-bounded sets. As a consequence, we get a stronger ideal condition for $\mathcal{P}_{\mathcal{I}}$ : the composition of an $\mathcal{I}$-bounded $m$-homogeneous polynomial with any continuous homogeneous polynomial is $\mathcal{I}$ bounded. In Section 4 we compare the ideal of $\mathcal{I}$-bounded polynomials with the composition ideal of polynomials. We show that the two ideals coincide whenever $\mathcal{I}$ is surjective and satisfies Condition $\Gamma$ that is introduced in [3].

\section{§2. Preliminaries and notation}

In the following, $E, F$ and $Z$ denote arbitrary (complex or real) Banach spaces. Let $B_{E}$ denote the closed unit ball of $E$. If $x \in E$ and $\epsilon>0$ then $B_{\epsilon}(x)$ is the open ball of center $x$ and radius $\epsilon$.

We denote by $\mathcal{L}\left({ }^{m} E ; F\right)$ the space of all continuous $m$-linear mappings from $E \times \cdots \times E$ into $F$. Whenever $m=1, \mathcal{L}\left({ }^{1} E ; F\right)=\mathcal{L}(E ; F)$ coincides with the usual space of continuous linear operators, and for $m=0$ we agree that $\mathcal{L}\left({ }^{0} E ; F\right)$ is the space of constant mappings from $E$ to $F$ which is identified with $F$. A mapping $P: E \rightarrow F$ is a continuous $m$-homogeneous polynomial if there is $A \in \mathcal{L}\left({ }^{m} E ; F\right)$ such that $P(x)=A(x, \ldots, x)$ for all $x \in E$. Let $\mathcal{P}\left({ }^{m} E ; F\right)$ denote the space of 
all continuous $m$-homogeneous polynomials from $E$ to $F$, endowed with the usual sup norm. When $F$ is the scalar field we simply write $\mathcal{P}\left({ }^{m} E\right)$.

By $\widehat{\otimes}_{\pi}^{m, s} E$ and $\widehat{\otimes}_{\pi_{s}}^{m, s} E$ we denote the $m$-fold completed symmetric tensor product of $E$ endowed with the projective norm $\pi$ and the projective $s$-tensor norm $\pi_{s}$, respectively. The projective norm $\pi$ is well-known (see e.g. [20]) and the projective $s$-tensor norm $\pi_{s}$ is defined by

$$
\pi_{s}(z)=\inf \left\{\sum_{j=1}^{k}\left|\lambda_{j}\right|\left\|x_{j}\right\|^{m}: k \in \mathbb{N}, z=\sum_{j=1}^{k} \lambda_{j} x_{j} \otimes \cdots \otimes x_{j}\right\}
$$

for $z \in \otimes^{m, s} E$ (see [12]). It is worth pointing out that the projective norm $\pi$ induces a norm equivalent to the projective $s$-tensor norm $\pi_{s}$ on the $m$-fold symmetric tensor product.

Given $P \in \mathcal{P}\left({ }^{m} E ; F\right)$, we denote by $\check{P}$ the unique symmetric continuous $m$ linear map $\check{P} \in \mathcal{L}\left({ }^{m} E ; F\right)$ such that $\check{P}(x, \ldots, x)=P(x)$ for all $x \in E$. The $n$-th polarization constant of the Banach space $E$, denoted by $c(n, E)$, is

$$
c(n, E)=\inf \left\{C>0:\|\check{P}\| \leq C\|P\| \text { for all } P \in \mathcal{P}\left({ }^{n} E\right)\right\} .
$$

It is well known that

$$
1 \leq c(n, E) \leq \frac{m^{m}}{m !}
$$

for every Banach space $E$.

Also,

$$
\begin{aligned}
P_{L}: \widehat{\otimes}_{\pi}^{n, s} E \rightarrow F, & P_{L}(x \otimes \cdots \otimes x)=P(x), \\
P_{L, s}: \widehat{\otimes}_{\pi_{s}}^{n, s} E \rightarrow F, & P_{L, s}(x \otimes \cdots \otimes x)=P(x)
\end{aligned}
$$

denote the linearization of $P$. If we consider the map $\delta_{m}^{E}: E \rightarrow \otimes^{m, s} E$ given by $\delta_{m}^{E}(x)=x \otimes \cdots \otimes x$ it is clear that $P=P_{L} \circ \delta_{m}^{E}=P_{L, s} \circ \delta_{m}^{E}$. The map $\delta_{m}^{E}$ is continuous when $\otimes^{m, s} E$ is endowed with either $\pi$ or $\pi_{s}$. It is well-known that $\left\|P_{L, s}\right\|=\|P\|$ and $\left\|P_{L}\right\|=\|\check{P}\|$. For the general theory of homogeneous polynomials and symmetric tensor products we refer to [11] and [12].

An ideal of homogeneous polynomials (or polynomial ideal) $\mathcal{Q}$ is a subclass of the class of all continuous homogeneous polynomials between Banach spaces such that for all $n \in \mathbb{N}$ and Banach spaces $E$ and $F$, the components $\mathcal{Q}\left({ }^{n} E, F\right)=$ $\mathcal{P}\left({ }^{n} E, F\right) \cap \mathcal{Q}$ satisfy:

(i) $\mathcal{Q}\left({ }^{n} E, F\right)$ is a linear subspace of $\mathcal{P}\left({ }^{n} E, F\right)$ which contains the $n$-homogeneous polynomials of finite type.

(ii) The ideal property: if $u \in \mathcal{L}(G, E), P \in \mathcal{Q}\left({ }^{n} E, F\right)$ and $t \in \mathcal{L}(F, H)$, then the composition $t \circ P \circ u$ is in $\mathcal{Q}\left({ }^{n} G, H\right)$. 
If $\|\cdot\|_{\mathcal{Q}}: \mathcal{Q} \rightarrow \mathbb{R}^{+}$satisfies

$\left(\mathrm{i}^{\prime}\right)\left(\mathcal{Q}\left({ }^{n} E ; F\right),\|\cdot\|_{\mathcal{Q}}\right)$ is a normed (Banach) space for all $E, F$ and $n$,

(ii') $\left\|P^{n}: \mathbb{K} \rightarrow \mathbb{K}: P^{n}(x)=x^{n}\right\|_{\mathcal{Q}}=1$ for all $n$, and

(iii') if $u \in \mathcal{L}(G, E), P \in \mathcal{Q}\left({ }^{n} E, F\right)$ and $t \in \mathcal{L}(F, H)$, then $\|t \circ P \circ u\|_{\mathcal{Q}} \leq$ $\|t\|\|P\|_{\mathcal{Q}}\|u\|^{n}$

then $\left[\mathcal{Q},\|\cdot\|_{\mathcal{Q}}\right]$ is called a normed (Banach) polynomial ideal.

The case $n=1$ recovers the classical theory of (normed, Banach) operator ideals, for which the reader is referred to [7] and [9]. In particular we will use the notation $[\mathcal{I}, \iota]$ for an arbitrary normed operator ideal, that is, $\iota: \mathcal{I} \rightarrow \mathbb{R}^{+}$satisfies (i'), (ii') and (iii') above.

The polynomial ideal $\mathcal{Q}$ is said to be closed if each component $\mathcal{Q}\left({ }^{n} E ; F\right)$ is a closed subspace of $\mathcal{P}\left({ }^{n} E ; F\right)$.

An operator ideal $\mathcal{I}$ is surjective if $T$ belongs to $\mathcal{I}(E ; F)$ whenever $T \circ S \in$ $\mathcal{I}(Z ; F)$ for every surjection $S \in \mathcal{L}(Z ; E)$. Here, $E, F$ and $Z$ are arbitrary Banach spaces. Equivalently, $\mathcal{I}$ is surjective if $T$ belongs to $\mathcal{I}(E ; F)$ whenever $T\left(B_{E}\right) \subset$ $U\left(B_{Z}\right)$ for some $U \in \mathcal{I}(Z ; F)$ (see [22]).

Recall that, given a normed operator ideal $[\mathcal{I}, \iota]$, there is a smallest surjective operator ideal $\mathcal{I}^{\text {sur }}$ which contains $\mathcal{I}$. Moreover, a continuous linear operator $T \in$ $\mathcal{L}(E ; F)$ belongs to $\mathcal{I}^{\text {sur }}(E ; F)$ if and only if $T \circ Q_{E} \in \mathcal{I}$, where $Q_{E}: \ell_{1}\left(B_{E}\right) \rightarrow E$ is the canonical surjection. If we define $\iota^{\text {sur }}(T):=\iota\left(T \circ Q_{E}\right)$ then $\left[\mathcal{I}^{\text {sur }}, \iota^{\text {sur }}\right]$ is a normed operator ideal, which is Banach whenever $[\mathcal{I}, \iota]$ is (cf. [7, Section 9.8]). The ideal $\left[\mathcal{I}^{\text {sur }}, \iota^{\text {sur }}\right]$ is called the surjective hull of $[\mathcal{I}, \iota]$.

Given an operator ideal $[\mathcal{I}, \iota]$, the composition ideal of polynomials, $\mathcal{I} \circ \mathcal{P}$, consists of all homogeneous polynomials $P$ between Banach spaces that can be factored as $P=T \circ Q$ where $Q$ is a homogeneous polynomial and $T$ is a linear operator belonging to $\mathcal{I}$. For $m \in \mathbb{N}$ and Banach spaces $E$ and $F$, the usual composition norm $\|\cdot\|_{\mathcal{I} \circ \mathcal{P}}$ of an $m$-homogeneous polynomial $P \in \mathcal{I} \circ \mathcal{P}\left({ }^{m} E ; F\right)$ is given by

$$
\|P\|_{\mathcal{I} \circ \mathcal{P}}:=\inf \left\{\iota(T)\|Q\|: P=T \circ Q, Q \in \mathcal{P}\left({ }^{m} E ; G\right), T \in \mathcal{I}(G ; F)\right\},
$$

With this norm, $\mathcal{I} \circ \mathcal{P}$ becomes a Banach polynomial ideal whenever $[\mathcal{I}, \iota]$ is a Banach operator ideal (see [6]).

Let $E$ be a Banach space, and $1 \leq p, p^{\prime} \leq \infty$ be conjugate exponents. We recall that a subset $K$ of $E$ is said to be relatively p-compact if for some sequence $\left(x_{n}\right) \in \ell_{p}(E), K \subset\left\{\sum_{n} a_{n} x_{n} \mid\left(a_{n}\right) \in B_{\ell_{p}^{\prime}}\right\}$, where $\ell_{p}(E)$ denotes the space of $p$-summable sequences in $E$ endowed with its natural norm and $B_{\ell_{p}^{\prime}}$ denotes the closed unit ball of $\ell_{p}^{\prime}$. An operator $T \in \mathcal{L}(E ; F)$ is p-compact if $T\left(B_{E}\right)$ is relatively 
$p$-compact. In this case the norm $k_{p}$ is defined as

$$
k_{p}(T)=\inf \left\{\left\|\left(x_{n}\right)_{n}\right\|_{p}\right\},
$$

where the infimum is taken over all sequences $\left(x_{n}\right)_{n} \in \ell_{p}(F)$ fulfilling the above condition. The norm $k_{p}$ was introduced by Sinha and Karn [21] and characterized by Delgado, Piñeiro and Serrano [8, Proposition 3.15]. We shall use $\left[\mathcal{K}_{p}, k_{p}\right]$ to denote the Banach ideal formed by all $p$-compact operators. Pietsch [17] has recently revisited the theory of $p$-compact operators by using the general theory of operator ideals as developed in his monograph [15].

\section{§3. The $\mathcal{I}$-ideal of homogeneous polynomials}

Let $[\mathcal{I}, \iota]$ be a normed operator ideal, and let $F$ be a Banach space. Let $C_{\mathcal{I}}(F)$ stand for the collection of subsets $A \subset F$ such that $A \subset T\left(B_{Z}\right)$ for some Banach space $Z$ and some $T \in \mathcal{I}(Z ; F)$. We will call any $A \in C_{\mathcal{I}}(F)$ an $\mathcal{I}$-bounded set.

Notice that $\mathcal{I}$-bounded sets are bounded. The family $C_{\mathcal{I}}(F)$ was first considered in [22] (see also [13]). The central role that it plays in our work justifies our coining the term ' $\mathcal{I}$-bounded set' to name its elements.

Example 3.1. Let $E$ be a Banach space and $A$ be a subset of $E$. Then $A \in$ $C_{\mathcal{K}_{p}}(E)$ if and only if $A$ is relatively $p$-compact.

Proof. The necessity follows from the definition of $C_{\mathcal{K}_{p}}(E)$.

Conversely, if $A$ is relatively $p$-compact then it is bounded. Hence, the operator $T: \ell_{1}(A) \rightarrow E$ given by $T\left(\left(t_{x}\right)_{x \in A}\right):=\sum_{x \in A} t_{x} x$ is well-defined. As the closed absolutely convex hull of a relatively $p$-compact set is relatively $p$-compact, $\bar{\Gamma}(A)$ is relatively $p$-compact. Since $\bar{\Gamma}(A)=T\left(B_{\ell_{1}(A)}\right), T$ belongs to $\mathcal{K}_{p}\left(\ell_{1}(A) ; E\right)$. From the inclusion

$$
A \subset \bar{\Gamma}(A)=T\left(B_{\ell_{1}(A)}\right)
$$

we conclude that $A \in C_{\mathcal{K}_{p}}(E)$.

The following well-known property of $C_{\mathcal{I}}$ will allow us to define the $\mathcal{I}$-ideal of homogeneous polynomials: If $A_{1}, \ldots, A_{n} \in C_{\mathcal{I}}(F)$ and $t_{1}, \ldots, t_{n}$ are scalars, then $\bigcup_{i=1}^{n} A_{i}$ and $\sum_{i=1}^{n} t_{i} A_{i}$ belong to $C_{\mathcal{I}}(F)$ (see [13, Proposition 3]).

If $T \in \mathcal{L}(E ; F)$ is such that $T\left(B_{E}\right)$ belongs to $C_{\mathcal{I}}(F)$ then it cannot be concluded that $T \in \mathcal{I}(E ; F)$ in general. This condition actually characterizes surjective operator ideals. We omit the easy proof.

Proposition 3.2. Let $\mathcal{I}$ be an operator ideal. The following assertions are equivalent: 
(i) $\mathcal{I}$ is surjective.

(ii) For any Banach spaces $E$ and $F$ and any $T \in \mathcal{L}(E ; F), T \in \mathcal{I}(E ; F)$ if and only if $T\left(B_{E}\right) \in C_{\mathcal{I}}(F)$.

We shall say that an $m$-homogeneous polynomial $P \in \mathcal{P}\left({ }^{m} E ; F\right)$ is locally $\mathcal{I}$-bounded (or $\mathcal{I}$-bounded for short) if for every $x \in E$ there exists a neighborhood $V_{x}$ of $x$ such that $P\left(V_{x}\right) \in C_{\mathcal{I}}(F)$. The set of all locally $\mathcal{I}$-bounded $m$ homogeneous polynomials from $E$ to $F$ is denoted by $\mathcal{P}_{\mathcal{I}}\left({ }^{m} E ; F\right)$. When $m=1$ we write $\mathcal{L}_{\mathcal{I}}(E ; F)=\mathcal{P}_{\mathcal{I}}\left({ }^{1} E ; F\right)$. It is easy to prove that $P \in \mathcal{P}\left({ }^{m} E ; F\right)$ is $\mathcal{I}$-bounded if and only if $P\left(B_{E}\right) \in C_{\mathcal{I}}(F)$.

It is clear that $\mathcal{P}_{\mathcal{I}}\left({ }^{m} E ; F\right)$ is a vector space. Let us endow it with a norm. Given $P \in \mathcal{P}_{\mathcal{I}}\left({ }^{m} E ; F\right)$, we have $P\left(B_{E}\right) \subset T\left(B_{Z}\right)$ for some Banach space $Z$ and some $T \in \mathcal{I}(Z ; F)$. Define

$$
\|P\|_{\mathcal{I}}:=\inf \iota(T)
$$

where $T$ varies among those operators in $\mathcal{I}$ fulfilling the above condition.

Notice that

$$
\mathcal{I}(E ; F) \subset \mathcal{L}_{\mathcal{I}}(E ; F) \subset \mathcal{L}(E ; F)
$$

and

$$
\|T\| \leq\|T\|_{\mathcal{I}} \leq \iota(T)
$$

for all $T \in \mathcal{I}(E ; F)$.

By Proposition 3.2, it follows that $\mathcal{I}(E ; F)=\mathcal{L}_{\mathcal{I}}(E ; F)$ if and only if $\mathcal{I}$ is surjective.

Proposition 3.3. $\left[\mathcal{P}_{\mathcal{I}},\|\cdot\|_{\mathcal{I}}\right]$ is a normed ideal of polynomials.

Proof. The only condition that needs some attention is the ideal property. Let $P \in \mathcal{P}_{\mathcal{I}}\left({ }^{m} E ; F\right), u \in \mathcal{L}(G ; E)$ and $v \in \mathcal{L}(F ; Z)$. Clearly $v \circ P \in \mathcal{P}_{\mathcal{I}}\left({ }^{m} E ; Z\right)$ and $\|v \circ P\|_{\mathcal{I}} \leq\|v\|\|P\|_{\mathcal{I}}$.

On the other hand,

$$
P\left(u\left(B_{G}\right)\right) \subset P\left(\|u\| B_{E}\right)=\|u\|^{m} P\left(B_{E}\right) \subset\|u\|^{m} T\left(B_{H}\right)
$$

for some Banach space $H$ and some $T \in \mathcal{I}(H ; F)$. Then

$$
\|P \circ u\|_{\mathcal{I}} \leq \iota\left(\|u\|^{m} T\right)=\|u\|^{m} \iota(T) .
$$

Taking the infimum we get

$$
\|P \circ u\|_{\mathcal{I}} \leq\|u\|^{m}\|P\|_{\mathcal{I}} .
$$

Theorem 3.4. $\left[\mathcal{P}_{\mathcal{I}},\|\cdot\|_{\mathcal{I}}\right]$ is a Banach ideal of polynomials whenever $[\mathcal{I}, \iota]$ is a Banach ideal of operators. 
Proof. It suffices to prove that any absolutely convergent series in $\mathcal{P}_{\mathcal{I}}\left({ }^{m} E ; F\right)$ is convergent. Let $\left(P_{n}\right)_{n}$ be a sequence of polynomials in $\mathcal{P}_{\mathcal{I}}\left({ }^{m} E ; F\right)$ such that $M:=\sum_{n=0}^{\infty}\left\|P_{n}\right\|_{\mathcal{I}}<\infty$. As the induced usual norm $\|\cdot\|$ in $\mathcal{P}_{\mathcal{I}}\left({ }^{m} E ; F\right)$ is weaker than $\|\cdot\|_{\mathcal{I}}$ and $\left(\mathcal{P}\left({ }^{m} E ; F\right),\|\cdot\|\right)$ is complete it follows that $\sum_{n=0}^{\infty} P_{n}$ converges to some $P \in \mathcal{P}\left({ }^{m} E ; F\right)$. Let us prove that $P \in \mathcal{P}_{\mathcal{I}}\left({ }^{m} E ; F\right)$.

Let $\epsilon>0$. By the definition of $\|\cdot\|_{\mathcal{I}}$, for each $n$ there is a Banach space $Z_{\epsilon}^{n}$ and an operator $T_{\epsilon}^{n} \in \mathcal{I}\left(Z_{\epsilon}^{n} ; F\right)$ such that

$$
\left\|P_{n}\right\|_{\mathcal{I}}+\epsilon / 2^{n}>\iota\left(T_{\epsilon}^{n}\right)
$$

and

$$
P_{n}\left(B_{E}\right) \subset T_{\epsilon}^{n}\left(B_{\epsilon}^{n}\right)
$$

where $B_{\epsilon}^{n}$ denotes the closed unit ball of $Z_{\epsilon}^{n}$. Let

$$
\Lambda_{1}\left(\left(Z_{\epsilon}^{n}\right)_{n}\right):=\left\{\left(z_{n}\right)_{n} \in \Pi_{n} Z_{\epsilon}^{n}: p_{r}\left(\left(z_{n}\right)_{n}\right)=\sum_{n=0}^{\infty} r^{n}\left\|z_{n}\right\|<\infty \text { for all } 0<r<1\right\}
$$

endowed with the topology generated by the family of seminorms $\left\{p_{r}: 0<r<1\right\}$.

Let

$$
D:=\left\{\left(z_{n}\right)_{n} \in \Lambda_{1}\left(\left(Z_{\epsilon}^{n}\right)_{n}\right): z_{n} \in B_{\epsilon}^{n}\right\} .
$$

Denote by $Z$ the linear span of $D$ endowed with its Minkowski functional. Then $Z$ is a Banach space and $Z=\bigcup_{\lambda>0} \lambda D$. Define the map $T: Z \rightarrow F$ by $T\left(\lambda\left(z_{n}\right)_{n}\right):=$ $\lambda \sum_{n=0}^{\infty} T_{\epsilon}^{n}\left(z_{n}\right)$ for any $\lambda>0$ and any $\left(z_{n}\right)_{n} \in D$. From the linearity of each $T_{\epsilon}^{n}$ it follows that $T\left(\lambda\left(z_{n}\right)_{n}\right)=T\left(\lambda^{\prime}\left(z_{n}^{\prime}\right)_{n}\right)$ whenever $\lambda\left(z_{n}\right)_{n}=\lambda^{\prime}\left(z_{n}^{\prime}\right)_{n}$. By $(3.1)$,

$$
\sum_{n=0}^{\infty}\left\|T_{\epsilon}^{n}\left(z_{n}\right)\right\| \leq \sum_{n=0}^{\infty}\left\|T_{\epsilon}^{n}\right\| \leq \sum_{n=0}^{\infty} \iota\left(T_{\epsilon}^{n}\right) \leq \sum_{n=0}^{\infty}\left\|P_{n}\right\|_{\mathcal{I}}+\epsilon=M+\epsilon<\infty .
$$

Thus $T$ is well-defined, and clearly it is linear and continuous.

We now prove that $T \in \mathcal{I}(Z ; F)$. For each $n$ define $S_{n}: Z \rightarrow F$ by $S_{n}\left(\lambda\left(z_{j}\right)_{j}\right)$ $=\lambda T_{\epsilon}^{n}\left(z_{n}\right)$ for $\lambda>0$ and $\left(z_{n}\right)_{n} \in D$. If $\pi_{n}: Z \rightarrow Z_{n}$ denotes the projection on the $n$th coordinate, $S_{n}=T_{\epsilon}^{n} \circ \pi_{n}$. Then $S_{n} \in \mathcal{I}(Z ; F)$ by the ideal property. Since

$$
T\left(\left(z_{j}\right)_{j}\right)=\sum_{n=0}^{\infty} T_{\epsilon}^{n}\left(z_{n}\right)=\sum_{n=0}^{\infty} S_{n}\left(\left(z_{j}\right)_{j}\right)
$$

and

$$
\sum_{n=0}^{\infty} \iota\left(S_{n}\right) \leq \sum_{n=0}^{\infty} \iota\left(T_{\epsilon}^{n}\right)<\sum_{n=0}^{\infty}\left(\left\|P_{n}\right\|_{\mathcal{I}}+\epsilon / 2^{n}\right)=M+\epsilon<\infty
$$

it follows from the hypothesis of $[\mathcal{I}, \iota]$ being Banach that $T=\sum_{n=0}^{\infty} S_{n}$ belongs to $\mathcal{I}(Z ; F)$. 
Let us see that $P\left(B_{E}\right) \subset T(D)$. Given $x \in B_{E}$, by (3.2) for each $n$ there is $z_{n} \in B_{\epsilon}^{n}$ such that $P_{n}(x)=T_{\epsilon}^{n}\left(z_{n}\right)$. Then

$$
P(x)=\sum_{n=0}^{\infty} P_{n}(x)=\sum_{n=0}^{\infty} T_{\epsilon}^{n}\left(z_{n}\right)=T\left(\left(z_{n}\right)_{n}\right) .
$$

As $\left(z_{n}\right)_{n} \in D$, it follows that $P\left(B_{E}\right) \subset T(D)$.

We have proved that $P=\sum_{n=0}^{\infty} P_{n}$ belongs to $\mathcal{P}_{\mathcal{I}}\left({ }^{m} E ; F\right)$.

Given $T \in \mathcal{L}(E ; F)$, let $\otimes_{m} T: \hat{\otimes}_{\pi_{s}}^{m, s} E \rightarrow \hat{\otimes}_{\pi}^{m, s} F$ be defined by

$$
\otimes_{m} T\left(\sum_{i=1}^{n} \alpha_{i} x_{i} \otimes \cdots \otimes x_{i}\right)=\sum_{i=1}^{n} \alpha_{i} T\left(x_{i}\right) \otimes \cdots \otimes T\left(x_{i}\right)
$$

and extended by continuity to the completions. An operator ideal $[\mathcal{I}, \iota]$ is stable under the formation of symmetric tensor products if $\otimes_{m} T: \hat{\otimes}_{\pi_{s}}^{m, s} E \rightarrow \hat{\otimes}_{\pi}^{m, s} F$ belongs to $\mathcal{I}\left(\hat{\otimes}_{\pi_{s}}^{m, s} E ; \hat{\otimes}_{\pi}^{m, s} F\right)$ whenever $T \in \mathcal{I}(E ; F)$ and, in this case, $\iota\left(\otimes_{m} T\right) \leq$ $C \iota(T)^{m}$ for some positive constant $C$.

In [3, Theorem 3.4] the authors prove that the ideal of $p$-compact operators is stable under the formation of symmetric tensor products.

Theorem 3.5. If an operator ideal $[\mathcal{I}, \iota]$ is stable under the formation of symmetric tensor products then any continuous m-homogeneous polynomial maps $\mathcal{I}$-bounded sets to $\mathcal{I}$-bounded sets.

Proof. Let $P \in \mathcal{P}\left({ }^{m} E ; F\right)$ and $A \in C_{\mathcal{I}}(E)$. Then $A \subset T\left(B_{Z}\right)$ for some Banach space $Z$ and some $T \in \mathcal{I}(Z ; E)$. Using the commutativity of the diagram

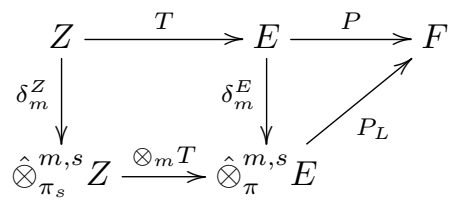

we get

$$
\begin{aligned}
P(A) & \subset P \circ T\left(B_{Z}\right)=P_{L} \circ \delta_{m}^{E} \circ T\left(B_{Z}\right)=P_{L} \circ\left(\otimes_{m} T\right) \circ \delta_{m}^{Z}\left(B_{Z}\right) \\
& \subset P_{L} \circ\left(\otimes_{m} T\right)\left(B_{\hat{\otimes}_{\pi_{s}}^{m, s} Z}\right) .
\end{aligned}
$$

By the stability assumption and the ideal property, $P_{L} \circ\left(\otimes_{m} T\right) \in \mathcal{I}\left(\hat{\otimes}_{\pi_{s}}^{m, s} Z ; F\right)$. Hence $P(A) \in C_{\mathcal{I}}(F)$.

As a consequence we get a stronger ideal condition for $\mathcal{P}_{\mathcal{I}}$. 
Theorem 3.6. Let $\mathcal{I}$ be an operator ideal and let $E, F, G$ and $Z$ be Banach spaces.

(i) If $P \in \mathcal{P}_{\mathcal{I}}\left({ }^{m} E ; F\right)$ and $Q \in \mathcal{P}\left({ }^{l} Z ; E\right)$ then $P \circ Q \in \mathcal{P}_{\mathcal{I}}\left({ }^{m l} Z ; F\right)$. In this case,

$$
\|P \circ Q\|_{\mathcal{I}} \leq\|P\|_{\mathcal{I}}\|Q\|^{m} .
$$

(ii) If $\mathcal{I}$ is stable under the formation of symmetric tensor products then $R \circ P \in$ $\mathcal{P}_{\mathcal{I}}\left({ }^{m k} E ; G\right)$ for any $P \in \mathcal{P}_{\mathcal{I}}\left({ }^{m} E ; F\right)$ and any $R \in \mathcal{P}\left({ }^{k} F ; G\right)$. In this case, there exists $K>0$ such that

$$
\|R \circ P\|_{\mathcal{I}} \leq K c(k, F)\|R\|\|P\|_{\mathcal{I}}^{m},
$$

where $c(k, F)$ is the $k$-th polarization constant of $F$.

Proof. (i) Let $T \in \mathcal{I}(H ; F)$ be so that $P\left(B_{E}\right) \subset T\left(B_{H}\right)$. Since $Q\left(B_{Z}\right) \subset\|Q\| B_{E}$,

$$
P \circ Q\left(B_{Z}\right) \subset P\left(\|Q\| B_{E}\right)=\|Q\|^{m} P\left(B_{E}\right) \subset\|Q\|^{m} T\left(B_{H}\right) .
$$

Then $\left.P \circ Q \in \mathcal{P}_{\mathcal{I}}{ }^{m l} Z ; F\right)$ and $\|P \circ Q\|_{\mathcal{I}} \leq\|Q\|^{m} \iota(T)$. Taking the infimum over $T$ we conclude that $\|P \circ Q\|_{\mathcal{I}} \leq\|P\|_{\mathcal{I}}\|Q\|^{m}$.

(ii) As $P \in \mathcal{P}_{\mathcal{I}}\left({ }^{m} E ; F\right)$, we have $P\left(B_{E}\right) \in C_{\mathcal{I}}(F)$. By Theorem $3.5, R\left(P\left(B_{E}\right)\right)$ $\in C_{\mathcal{I}}(G)$. Then $R \circ P \in \mathcal{P}_{\mathcal{I}}\left({ }^{m k} E ; G\right)$. However, in order to get the inequality for the norms, the above argument needs to be more precise: Let $T \in \mathcal{I}(H ; F)$ be so that $P\left(B_{E}\right) \subset T\left(B_{H}\right)$. Taking $A=P\left(B_{E}\right)$ in the proof of Theorem 3.5, we get

$$
R \circ P\left(B_{E}\right) \subset R_{L} \circ\left(\otimes_{m} T\right)\left(B_{\hat{\otimes}_{\pi_{s}}^{m, s} H}\right) .
$$

Since $\otimes_{m} T \in \mathcal{I}\left(\hat{\otimes}_{\pi_{s}}^{m, s} H ; \hat{\otimes}_{\pi}^{m, s} F\right)$ then

$$
\|R \circ P\|_{\mathcal{I}} \leq \iota\left(R_{L} \circ\left(\otimes_{m} T\right)\right) \leq\left\|R_{L}\right\| \iota\left(\otimes_{m} T\right) \leq c(k, F)\|R\| K \iota(T)^{m},
$$

for a suitable $K>0$. As $T$ is arbitrary,

$$
\|R \circ P\|_{\mathcal{I}} \leq K c(k, F)\|R\|\|P\|_{\mathcal{I}}^{m} .
$$

\section{$\S 4$. Comparing with the composition ideal of polynomials}

An operator ideal $\mathcal{I}$ is said to satisfy Condition $\Gamma$ if the closed absolutely convex hull of any $\mathcal{I}$-bounded set is $\mathcal{I}$-bounded. Given a subset $A$ of a Banach space $E$, the closed absolutely convex hull of $A$ is denoted by $\bar{\Gamma}(A)$.

Example 4.1. By [13, Proposition 3] any operator ideal which is surjective and closed satisfies Condition $\Gamma$. The most usual examples are the ideals of compact operators and weakly compact operators. 
Example 4.2. For $1<p<\infty$, the ideal $\left[K_{p}, k_{p}\right]$ of all $p$-compact operators is surjective and satisfies Condition $\Gamma$, although $K_{p}$ is not closed (see $[2,3]$ ).

Let us consider the surjective hull $\mathcal{N}^{\text {sur }}$ of the ideal $\mathcal{N}$ of nuclear operators. By [7, Ex. 9.14], $T \in \mathcal{L}(E ; F)$ belongs to $\mathcal{N}^{\text {sur }}(E ; F)$ if and only if there is a sequence $\left(y_{n}\right)_{n} \in \ell_{1}(F)$ such that

$$
T\left(B_{E}\right) \subset\left\{\sum_{n=1}^{\infty} \lambda_{n} y_{n}:\left|\lambda_{n}\right| \leq 1\right\} .
$$

This description shows that $\mathcal{N}^{\text {sur }}$ coincides with $K_{1}$, the ideal of 1-compact linear operators.

Example 4.3. The ideal $\mathcal{N}^{\text {sur }}$ satisfies Condition $\Gamma$.

Proof. Let $A \in C_{\mathcal{N}_{\text {sur }}}(F)$. Then there is a Banach space $E$ and an operator $T \in$ $\mathcal{N}^{\operatorname{sur}}(E ; F)$ such that $A \subset T\left(B_{E}\right)$. Choose a sequence $\left(y_{n}\right)_{n} \in \ell_{1}(F)$ such that

$$
T\left(B_{E}\right) \subset\left\{\sum_{n=1}^{\infty} \lambda_{n} y_{n}:\left|\lambda_{n}\right| \leq 1\right\} .
$$

Consider the nuclear map $\phi_{\left(y_{n}\right)_{n}}: \ell^{\infty} \rightarrow F$ defined by $\phi_{\left(y_{n}\right)_{n}}\left(\left(\lambda_{n}\right)_{n}\right)=\sum_{n=1}^{\infty} \lambda_{n} y_{n}$. Notice that

$$
\phi_{\left(y_{n}\right)_{n}}\left(B_{\ell}\right)=\left\{\sum_{n=1}^{\infty} \lambda_{n} y_{n}:\left|\lambda_{n}\right| \leq 1\right\} .
$$

As $\phi_{\left(y_{n}\right)_{n}}$ is linear, $\phi_{\left(y_{n}\right)_{n}}\left(B_{\ell^{\infty}}\right)$ is absolutely convex. Let us see that $\phi_{\left(y_{n}\right)_{n}}$ is weak* ${ }^{*}$-norm continuous. Let $\epsilon>0$. The set

$$
V:=\left\{\left(\lambda_{n}\right)_{n} \in \ell^{\infty}: \sum_{n=1}^{\infty}\left|\lambda_{n}\right|\left\|y_{n}\right\|<\epsilon\right\}
$$

is a weak* neighbourhood of 0 . Since $\phi_{\left(y_{n}\right)_{n}}(V) \subset \epsilon B_{F}, \phi_{\left(y_{n}\right)_{n}}$ is weak*-norm continuous.

Since $B_{\ell^{\infty}}$ is weak* compact, we see that $\phi_{\left(y_{n}\right)_{n}}\left(B_{\ell^{\infty}}\right)$ is norm compact in $F$. Hence,

$$
\bar{\Gamma}(A) \subset \bar{\Gamma}\left(\phi_{\left(y_{n}\right)_{n}}\left(B_{\ell^{\infty}}\right)\right)=\phi_{\left(y_{n}\right)_{n}}\left(B_{\ell^{\infty}}\right) .
$$

Since $\phi_{\left(y_{n}\right)_{n}}$ trivially belongs to $\mathcal{N}^{\text {sur }}\left(\ell^{\infty} ; F\right)$, we conclude that $\bar{\Gamma}(A) \in C_{\mathcal{N}^{\text {sur }}}(F)$.

Other examples of surjective (not necessarily closed) operator ideals fulfilling Condition $\Gamma$ can be easily constructed by considering the surjective hull of an operator ideal that enjoys Condition $\Gamma$, as is shown in the following result. 
Proposition 4.4. If an operator ideal $\mathcal{I}$ satisfies Condition $\Gamma$ then $\mathcal{I}^{\text {sur }}$ also satisfies Condition $\Gamma$.

Proof. Let $\mathcal{I}$ be an operator ideal satisfying Condition $\Gamma$, and let $F$ be a Banach space. Consider a set $A \subset F$ such that $A \in C_{\mathcal{I}^{\text {sur }}}(F)$. We have to prove that $\bar{\Gamma}(A) \in$ $C_{\mathcal{I}^{\text {sur }}}(F)$. Choose a Banach space $Z$ and $T \in \mathcal{I}^{\text {sur }}(Z ; F)$ such that $A \subset T\left(B_{Z}\right)$. By the characterization of the surjective hull, $T \circ Q_{Z} \in \mathcal{I}\left(\ell_{1}\left(B_{Z}\right) ; F\right)$. Since $B_{Z} \subset$ $Q_{Z}\left(B_{\ell_{1}\left(B_{Z}\right)}\right)$, it follows that $A \subset T\left(Q_{Z}\left(B_{\ell_{1}\left(B_{Z}\right)}\right)\right)$. Thus we see that $A$ belongs to $C_{\mathcal{I}}(F)$. Since $\mathcal{I}$ satisfies Condition $\Gamma, \bar{\Gamma}(A) \in C_{\mathcal{I}}(F)$. The inclusion $C_{\mathcal{I}}(F) \subset$ $C_{\mathcal{I}^{\text {sur }}}(F)$ yields the result.

Condition $\Gamma$ has been used strongly in [4], where it is essential when working with non-closed ideals of operators. Let us see that $\mathcal{P}_{\mathcal{I}}$ turns out to be the composition ideal of polynomials $\mathcal{I} \circ \mathcal{P}$ whenever $\mathcal{I}$ is a surjective ideal fulfilling Condition $\Gamma$.

Theorem 4.5. Let $P \in \mathcal{P}\left({ }^{m} E ; F\right)$.

(i) If $P_{L, s} \in \mathcal{L}_{\mathcal{I}}\left(\hat{\otimes}_{\pi_{s}}^{m, s} E ; F\right)$ then $P \in \mathcal{P}_{\mathcal{I}}\left({ }^{m} E ; F\right)$.

(ii) If $\mathcal{I}$ satisfies Condition $\Gamma$ then the converse to (i) holds: if $P \in \mathcal{P}_{\mathcal{I}}\left({ }^{m} E ; F\right)$ then $P_{L, s} \in \mathcal{L}_{\mathcal{I}}\left(\hat{\otimes}_{\pi_{s}}^{m, s} E ; F\right)$.

Proof. (i) If $P_{L, s} \in \mathcal{L}_{\mathcal{I}}\left(\hat{\otimes}_{\pi_{s}}^{m, s} E ; F\right)$ then $P_{L, s}\left(B_{\hat{\otimes}_{\pi_{s}}^{m, s} E}\right) \in C_{\mathcal{I}}(F)$. As $P\left(B_{E}\right) \subset$ $P_{L, s}\left(B_{\hat{\otimes}_{\pi_{s}}^{m, s} E}\right)$ it follows that $P\left(B_{E}\right)$ is $\mathcal{I}$-bounded.

(ii) Since $P\left(B_{E}\right)$ is $\mathcal{I}$-bounded, Condition $\Gamma$ ensures that $\bar{\Gamma}\left(P\left(B_{E}\right)\right)$ also is. The chain

$$
P_{L, s}\left(B_{\hat{\otimes}_{\pi_{s}}^{m, s} E}\right)=P_{L, s}\left(\bar{\Gamma}\left(\delta^{m}\left(B_{E}\right)\right)\right) \subset \bar{\Gamma}\left(P_{L, s}\left(\delta^{m}\left(B_{E}\right)\right)\right)=\bar{\Gamma}\left(P\left(B_{E}\right)\right)
$$

proves now that $P_{L, s}\left(B_{\hat{\otimes}_{\pi_{s}}^{m, s} E}\right)$ is $\mathcal{I}$-bounded too.

The next result partially recovers [3, Theorem 3.1] in the case of $p$-compact homogeneous polynomials.

Corollary 4.6. If $\mathcal{I}$ is surjective and satisfies Condition $\Gamma$ then $\mathcal{P}_{\mathcal{I}}\left({ }^{m} E ; F\right)=$ $\mathcal{I} \circ \mathcal{P}\left({ }^{m} E ; F\right)$ for all Banach spaces $E$ and $F$, and $\|\cdot\|_{\mathcal{I}} \leq\|\cdot\|_{\mathcal{I} \circ \mathcal{P}}$.

Proof. By Theorem 4.5, $P \in \mathcal{P}_{\mathcal{I}}\left({ }^{m} E ; F\right)$ if and only if $P_{L, s} \in \mathcal{L}_{\mathcal{I}}\left(\hat{\otimes}_{\pi_{s}}^{m, s} E ; F\right)$. By Proposition 3.2, $\mathcal{L}_{\mathcal{I}}\left(\hat{\otimes}_{\pi_{s}}^{m, s} E ; F\right)=\mathcal{I}\left(\hat{\otimes}_{\pi_{s}}^{m, s} E ; F\right)$. The equality $\mathcal{P}_{\mathcal{I}}\left({ }^{m} E ; F\right)=$ $\mathcal{I} \circ \mathcal{P}\left({ }^{m} E ; F\right)$ follows now from [6, Proposition 3.2]. Let $P \in \mathcal{P}_{\mathcal{I}}\left({ }^{m} E ; F\right)$. Then, by [6, Proposition 3.7],

$$
\|P\|_{\mathcal{I}} \leq\left\|P_{L, s}\right\|_{\mathcal{I}} \leq \iota\left(P_{L, s}\right)=\|P\|_{\mathcal{I} \circ \mathcal{P}} .
$$


Corollary 4.7. If $\mathcal{I}$ is closed and surjective then $\|P\|_{\mathcal{I}}=\|P\|$ for all $P \in$ $\mathcal{P}_{\mathcal{I}}\left({ }^{m} E ; F\right)$.

Proof. The inequality $\|P\| \leq\|P\|_{\mathcal{I}}$ always holds. If $\mathcal{I}$ is closed and surjective then by [13, Proposition 3], $\mathcal{I}$ satisfies Condition $\Gamma$. If $P \in \mathcal{P}_{\mathcal{I}}\left({ }^{m} E ; F\right)$, then by Theorem 4.5 and Proposition 3.2, $P_{L, s} \in \mathcal{L}_{\mathcal{I}}\left(\hat{\otimes}_{\pi_{s}}^{m, s} E ; F\right)=\mathcal{I}\left(\hat{\otimes}_{\pi_{s}}^{m, s} E ; F\right)$. Consequently,

$$
\|P\|_{\mathcal{I}} \leq \iota\left(P_{L, s}\right)=\left\|P_{L, s}\right\|=\|P\|
$$

for all $P \in \mathcal{P}_{\mathcal{I}}\left({ }^{m} E ; F\right)$.

\section{Acknowledgements}

This paper was started while the second author was visiting the Department of Mathematical Sciences at Kent State University. She thanks this Department for its kind hospitality. The authors were supported in part by MICINN (Project MTM2008-03211 and MTM2011-22417). The second author was also supported by Ministerio de Ciencia e Innovación, Programa Nacional de Movilidad de Recursos Humanos del Plan Nacional de I-D+i 2008-2011(MICINN Ref. PR2009-0042).

\section{References}

[1] R. M. Aron, G. Botelho, D. Pellegrino and P. Rueda, Holomorphic mappings associated to composition ideals of polynomials, Rend. Lincei Mat. Appl. 21 (2010), 261-274. Zbl 1204.46029 MR 2677604

[2] R. M. Aron, M. Maestre and M. P. Rueda, p-Compact holomorphic mappings, Rev. R. Acad. Ciencias Exactas Fís. Nat. Ser. A Mat. 104 (2010), 353-364. Zbl 1242.46055 MR 2757246

[3] R. M. Aron and P. Rueda, p-Compact homogeneous polynomials from an ideal point of view, in Function spaces in modern analysis, K. Jarosz (ed.), Contemp. Math. 547, Amer. Math. Soc., 2011, 61-71. Zbl 1238.46041 MR 2856480

[4] $\_$I-Bounded holomorphic functions, preprint.

[5] R. M. Aron and M. Schottenloher, Compact holomorphic mappings on Banach spaces and the approximation property, J. Funct. Anal. 21 (1976), 7-30. Zbl 0328.46046 MR 0402504

[6] G. Botelho, D. Pellegrino and P. Rueda, On composition ideals of multilinear mappings and homogeneous polynomials, Publ. RIMS Kyoto Univ. 43 (2007), 1139-1155. Zbl 1169.46023 MR 2389796

[7] A. Defant and K. Floret, Tensor norms and operator ideals, North-Holland Math. Stud. 176, North-Holland, 1993. Zbl 0774.46018 MR 1209438

[8] J. M. Delgado, C. Piñeiro and E. Serrano, Operators whose adjoints are quasi $p$-nuclear, Studia Math. 197 (2010), 291-304. Zbl 1190.47024 MR 2607494

[9] J. Diestel, H. Jarchow and A. Pietsch, Operator ideals, in Handbook of the geometry of Banach spaces, Vol. I, North-Holland, 2001, 437-496. Zbl 1012.47001 MR 1863699

[10] J. Diestel and J. J. Uhl, Jr., Vector measures, Math. Surveys Monogr. 15, Amer. Math. Soc., 1977. Z Zbl 0369.46039 MR 0453964

[11] S. Dineen, Complex analysis on infinite dimensional spaces, Springer, London, 1999. Zbl 1034.46504 MR 1705327 
[12] K. Floret, Natural norms on symmetric tensor products of normed spaces, Note Mat. 17 (1997), 153-188. Zbl 0961.46013 MR 1749787

[13] M. González and J. M. Gutiérrez, Surjective factorization of holomorphic mappings, Comment. Math. Univ. Carolin. 41 (2000), 469-476. Zbl 1040.46033 MR 1795078

[14] A. Persson and A. Pietsch, $p$-nukleare und p-integrale Abbildungen in Banachräumen, Studia Math. 33 (1969), 19-62. Zbl 0189.43602 MR 0243323

[15] A. Pietsch, Operator ideals, North-Holland Math. Library 20, North-Holland, Amsterdam, 1980. Zbl 0434.47030 MR 0582655

[16] _ Ideals of multilinear functionals (designs of a theory), in Proceedings of the second international conference on operator algebras, ideals and their applications in theoretical physics, Teubner-Texte Math. 67, Teubner, Leipzig, 1984, 185-199. Zbl 0562.47037 MR 0763541

[17] _ The ideal of $p$-compact operators and its maximal hull, Proc. Amer. Math. Soc., to appear.

[18] O. I. Reinov, Approximation properties of order $p$ and the existence of non- $p$-nuclear operators with $p$-nuclear second adjoints, Math. Nachr. 109 (1982), 125-134. MR 0705902

[19] R. Ryan, Applications of topological tensor products to infinite dimensional holomorphy, PhD thesis, Trinity College, 1980.

[20] _ Introduction to tensor products of Banach spaces, Springer Monogr. Math., Springer, London, 2002. Zbl 1090.46001 MR 1888309

[21] D. P. Sinha and A. K. Karn, Compact operators whose adjoints factor through subspaces of $\ell_{p}$, Studia Math. 150 (2002), 17-33. Zbl 1008.46008 MR 1893422

[22] I. Stephani, Generating systems of sets and quotients of surjective operator ideals, Math. Nachr. 99 (1980), 13-27. Zbl 0474.47019 MR 0637639 